\title{
TAGUNG
}

\section{Wie viel Freiheit opfert die EU für unsere Sicherheit?}

\author{
Frédéric Krumbein*
}

Die Tagung „Rechtsstaat, Freiheit und Sicherheit in Europa“" wurde von der Andrássy Universität in Budapest, der Societas Iuris Publici Europaei und dem Arbeitskreis Europäische Integration e.V. organisiert. Die zweitägige Konferenz beschäftigte sich im Wesentlichen mit der dritten Säule der Europäischen Union, dem Raum der Freiheit, der Sicherheit und des Rechts. Den Hintergrund der Tagung bildeten aktuelle Herausforderungen, wie der Kampf gegen den internationalen Terrorismus und die damit einhergehende Einschränkung von Grundrechten. Die Tagung gliederte sich in vier thematische Abschnitte: 1) Herausforderungen für Freiheit und Sicherheit, 2) Öffentliche Sicherheit und individuelle Freiheiten im nationalen Rechtsstaat, 3) Freiheit und Sicherheit in der globalen Sicherheitsarchitektur: die europäische Dimension und 4) Freiheit und Sicherheit in der globalen Sicherheitsarchitektur: die internationale Dimension.

Die grundlegende Frage der Tagung war: Wie viel Freiheit opfern wir für unsere Sicherheit? Das ewige Spannungsverhältnis zwischen Freiheit und Sicherheit stand somit im Vordergrund. Der 11. September 2001 wurde dabei als die Wasserscheide bezeichnet, welche das Verhältnis zugunsten der Sicherheit neu austariert hat.

Herausforderungen für Freiheit und Sicherheit

Otto Pfersmann befasste sich mit dem Begriff Sicherheit. Als erstes konstatierte er, dass nach dem 11. September 2001 viele Freiheiten schnell und häufig allzu leichtfertig geopfert wurden. Nach dem amerikanischen Philo-

\section{Rechtsstaat, Freiheit und Sicherheit in Europa}

Tagung der „Societas Iuris Publici Europaei“ (SIPE), der Andrássy Universität Budapest und des Arbeitskreises Europäische Integration e.V.

Budapest, 5./6. Juni 2009

Freiheit und Sicherheit am Anfang des 21. Jahrhunderts

Herausforderungen für Freiheit und Sicherheit Vorsitz

Prof. Dr. Antonio d'Atena, Rom

Der Wandel des Sicherheitsverständnisses im 21. Jahrhundert

Prof. Dr. Otto Pfersmann, Paris/Wien

Rechtsstaatliche Antworten der Demokratie auf ihre Gegner: nationale, supranationale und internationale Aspekte

Prof. Dr. Eivind Smith, Oslo

Öffentlichkeit und Transparenz des staatlichen Sicherheitshandelns als transnationales (Rechts-) Prinzip

Prof. Dr. Anne Peters, Basel

Öffentliche Sicherheit und individuelle Freiheiten im nationalen Rechtsstaat

Vorsitz

Prof. Dr. Pedro Cruz Villalón, Madrid

Öffentliche Sicherheit und Grundprinzipien des Strafrechts

Prof. Dr. Leonard Besselink, Utrecht

Recht auf Privatheit versus öffentliche Sicherheit Prof. Dr. Ciril Ribičič, Ljubljana

Meinungs- und Pressefreiheit versus öffentliche Sicherheit

Prof. Dr. Ricardo García Macho, Castelló de la Plana

Der transnationale Dialog der Gerichte in AntiTerrorismusfällen

Prof. Dr. Tania Groppi, Siena

Zusammenfassende Schlussbemerkungen Prof. Dr. Didier Maus, Paris

Frédéric Krumbein, Geschäftsführer des Arbeitskreises Europäische Integration e.V., Berlin. 
sophen John Rawls rechtfertigt sich ein Eingriff in die Freiheit nur, wenn dadurch mehr Freiheit für alle Bürger entsteht.

Sicherheit ist keine Norm, sondern ein Konzept. Verschiedene Konzepte lassen sich dabei unterscheiden. Erstens, es gibt eine Sicherheit des Staates (den Frieden). Zweitens, es gibt eine Sicherheit des Einzelnen, die sich vor allem im Gefühl von Sicherheit manifestiert (subjektive Sicherheit). Drittens, es gibt eine strukturelle Sicherheit (objektive Sicherheit). Thomas Hobbes hat Sicherheit als ,decent survival" des Individuums definiert. Dabei ging es nicht nur um die Sicherung des Überlebens, sondern auch um das Glück des Einzelnen.

Eine weitere Frage beim Konzept der Sicherheit ist die nach einem Grundrecht auf Sicherheit. Existiert ein solches Grundrecht? Wer setzt dieses durch? Ein solches Grundrecht existiert in Form des Menschenrechts auf Leben und Sicherheit der Person. Die Durchsetzung dieses Rechts muss dabei zunehmend auf der globalen Ebene erfolgen, um effektiv zu sein. Eine globale Durchsetzung existiert aber bislang nicht.

Eivind Smith referierte in seinem Vortrag über Demokratie und ihre Gegner. Die Demokratie als Regierungsform ist in den letzten Jahrzehnten weltweit expandiert. Ihre Hauptgegner beziehungsweise -bedrohungen sind ,failed states', Autokratien und gewalttätige Gruppen innerhalb ihrer eigenen Gesellschaft, welche die Demokratie als Regierungsform bedrohen. Eine grundlegende Frage ist dabei: Wie reagieren Demokratien auf ihre Gegner? Eine der wesentlichen Elemente der Demokratie ist die Meinungsfreiheit und die verbale Auseinandersetzung zwischen politischen Gegnern verbunden mit dem Verzicht der Anwendung von Gewalt. Wie kann die Demokratie vor diesem Hintergrund ihrer eigenen Werte auf Propaganda und auf illegale Gewalt reagieren? Soll jede politische Partei in einer Demokratie um politische Macht konkurrieren dürfen? Smith nennt hier das Mittel des
Freiheit und Sicherheit in der globalen Sicherheitsarchitektur

Die europäische Dimension

Vorsitz

Prof. Dr. Hartmut Bauer, Potsdam

Innere Sicherheit als europäische Aufgabe?

Prof. Dr. Franz Merli, Graz

Der europäische Haftbefehl in der Rechtsprechung nationaler Verfassungsgerichte und oberster Gerichte

Prof. Dr. Péter Kovács, Budapest

Auf dem Weg zu einer europäischen Rechtsprechung zum Raum der Freiheit, der Sicherheit und des Rechts

Prof. Dr. Eleanor Sharpston, Luxemburg

\section{Die internationale Dimension}

Vorsitz

Prof. Dr. Jean-François Flauss, Paris/Straßburg

Eingriffe von Sicherheitsbehörden und privaten Akteuren aus der Sicht des Völkerrechts Prof. Dr. Lauri Mälksoo, Tartu

Der verfassungsrechtliche Rahmen für Akte der Armee und der Sicherheitskräfte im Falle der Gefährdung der internationalen Sicherheit Prof. Dr. Xavier Philippe, Aix-en-Provence

Die Haltung der europäischen Gerichte zu Sicherheitsfragen

Prof. Dr. Heike Krieger, Berlin

Zusammenfassende Schlussbemerkungen Prof. Dr. Dieter Scheuing, Würzburg

Parteienverbots zur Ausgrenzung von Verfassungsfeinden, wie es zum Beispiel in Deutschland und der Türkei existiert. Eine weitere Frage ist, wie man mit Wahlerfolgen von Parteien umgeht, welche demokratiefeindlich sind beziehungsweise demokratische Werte infrage stellen. Beispiele hierfür sind der Wahlsieg der Hamas in Palästina oder die Freiheitliche Partei Österreichs (FPÖ) in Österreich unter ihrem zwischenzeitlich verstorbenen Vorsitzenden Jörg Haider. Wo zieht man in solchen Fällen die Grenze? In der Türkei werden die gemäßigt islamistische Regierungspartei AKP (Gerechtigkeits- und Entwicklungspartei) und kurdische Parteien von Teilen der Elite als potenziell systembedrohend angesehen. Was sind illegale gewalttätige Gruppen? Die Terroris- 
ten des einen sind oft die Freiheitskämpfer des anderen und umgekehrt.

Eine weitere Frage ist die nach den Grenzen der Meinungsfreiheit. Sollte die Leugnung des Holocaust und anderer Genozide (Beispiel Armenien) verboten werden? Die Gefahr besteht, dass einige Staaten solche Verbote als Vorwand zu weiter gehenden Einschränkungen der Meinungsfreiheit benutzen. Neben der Systembedrohung kann auch die Moral als Argument der Einschränkung der Freiheit dienen. Die Mohammedkarikaturen in Dänemark sind ein Beispiel für umstrittene Meinungsäußerungen, die unter dem Aspekt der Moral diskutiert werden könnten. Das Verbot bestimmter Meinungen ist oftmals nicht ausreichend. Es drängt extremistische Meinungen in Subkulturen ab. Jedes Verbot sollte daher auf seine Effizienz hin überprüft werden. Extremisten üben mit der Äußerung ihrer Meinungen und der Partizipation an freien Wahlen demokratische Grundfunktionen aus. Insofern stellt sich die Frage, ob sie wirklich Feinde der Demokratie sind. Die Antwort auf diese Frage hängt von der jeweiligen Gesellschaft ab und ob die Frage in jungen oder etablierten Demokratien gestellt wird. Zusammenfassend zieht Smith drei Schlussfolgerungen. Erstens, der Rechtsstaat ist wichtig. Er ist für die Verteidigung der Demokratie aber nicht ausreichend. Zweitens, Kritik ist ein entscheidender demokratischer Wert. Ein Beispiel ist der EU-Boykott von Österreich nach der Regierungsbeteiligung der FPÖ, den Smith als legitim ansieht. Jemand darf seine Meinung äußern (österreichisches Volk), aber die anderen (Europäische Union) dürfen dann das Gespräch verweigern und sich zurückziehen. Drittens, das Recht auf Widerstand ist ein legitimes Mittel der Verteidigung der Demokratie.

Anne Peters sprach anschließend in ihrem Vortrag über das schwierige Verhältnis zwischen Transparenz und Sicherheit. Transparenz kann eine Gefahr für die Sicherheit darstellen, aber auch die Sicherheit erhöhen. Transparenz meint dabei in erster Linie Of- fenheit und damit im rechtlichen und politischen Kontext den freien Zugang der Bürger zu Gesetzen, Regeln und Entscheidungen.

Die Entscheidung von US-Präsident Barack Obama keine weiteren Fotos von Folterungen durch US-Soldaten zu veröffentlichen kann zum Beispiel als eine Entscheidung für die Sicherheit und gegen die Transparenz verstanden werden. Transparenz selbst ist ein Ergebnis der Aufklärung und ein wichtiger Bestandteil von Demokratien. Die Öffentlichkeit von Parlamentsdebatten und damit die Öffentlichkeit des legislativen Prozesses war dabei lange umstritten. Eine neuere Form von Transparenz ist die Transparenz der Verwaltung (zum Beispiel bei Umweltverordnungen). Der Kampf gegen Korruption ist ebenfalls ein politisches Ziel vieler internationaler Organisationen und anderer entwicklungspolitischer Akteure als Teil der Förderung von ,Good Governance، geworden (Beispiel Weltbank). Transparenz hat einen instrumentellen Wert und keinen Wert an sich. Transparenz kann die Akzeptanz und Legitimität von Regierungen erhöhen. Die Effektivität und die Verantwortlichkeit von Handeln erhöht sich ebenfalls bei staatlichen und nichtstaatlichen Akteuren. Im rechtlichen Bereich steigert Transparenz die Vorhersehbarkeit und die rechtliche Sicherheit der Akteure. Gleichzeitig kann Transparenz aber auch negative Konsequenzen nach sich ziehen, wie Inflexibilität und sozialen Stress.

Gesetze, welche den Zugang der Bürger zu Informationen regeln, wurden in vielen Staaten erlassen, so zum Beispiel in Schweden (Freedom of the Press Act 1949), den USA (Freedom of Information Act 1966), Frankreich (Loi n ${ }^{\circ}$ 78-753 du 17 juillet 1978 de la liberté d'accès aux documents administratifs), Großbritannien (Freedom of Information Act 2000), Schweiz (Öffentlichkeitsgesetz 2004) und Deutschland (Informationsfreiheitsgesetz 2005). Die USA haben dabei eine Vorreiterrolle gespielt. Ausnahmen für Transparenz gab es schon immer im Bereich der inneren und äußeren Sicherheit, dem öffentlichen In- 
teresse und der Privatsphäre. Nach dem 11. September 2001 hat die Transparenz abgenommen. Die USA waren auch bei dieser Entwicklung der Vorreiter. In einigen Fällen kann von staatlichen Organen sogar verweigert werden darüber Auskunft zu geben, ob überhaupt Informationen zu einem Sachverhalt existieren (sogenannte ,Glomar Response' nach einem Gerichtsurteil in den USA von 2005). Im rechtlichen Bereich bilden die US-Militärtribunale und die Debatten um ihre Einrichtung und Verfahrensweisen einen aktuellen Fall der Abwägung zwischen den Prinzipien Freiheit und Sicherheit. Schließlich kann Transparenz aber auch als Erfüllung eines Menschenrechts auf Information angesehen werden. In den USA hat die Regierung nach Urteil von Gerichten die Verpflichtung ihren Bürgern den Zugang zu Informationen zu gewähren (mit legitimen Ausnahmen wie staatlichen Sicherheitsinteressen). Demokratietheoretisch kann man argumentieren, dass Informationen rechtmäßig den Bürgern gehören. Umgekehrt kann zuviel Transparenz diplomatische Verhandlungen und das Finden von Lösungen erschweren beziehungsweise die Entscheidungsprozesse in andere Institutionen (zum Beispiel Hinterzimmer) verlagern. Die Gegenposition zu geheimen Verhandlungen hat Präsident Woodrow Wilson vertreten, der im ersten seiner 14 Punkte von 1918 in der internationalen Diplomatie Öffentlichkeit und Transparenz forderte. Im Bereich der Nonproliferation von Massenvernichtungswaffen ist Transparenz ebenfalls ein fundamentales Prinzip. Die Weigerung des Irans vollständige Transparenz über seine nuklearen Aktivitäten herzustellen ist ein Grund für internationale Sanktionen.

\section{Öffentliche Sicherheit und individuelle Frei- heiten im nationalen Rechtsstaat}

Den ersten Vortrag hielt Leonard Besselink. Besselink hat das Strafrecht mehrerer europäischer Staaten verglichen. Dabei hat er nur zwei wesentliche gemeinsame Rechtsprinzipien festgestellt und zwar erstens, habeas corpus' und zweitens ,nulla poena sine lege'. Der
Grundsatz ,nulla poena sine lege " findet sich in allen Verfassungen der EU-Staaten, welche eine Verfassung besitzen (einzige Ausnahme: Großbritannien). Zwei aktuelle Tendenzen gibt es im Strafrecht. Erstens wird Prävention zunehmend bedeutender. Die kriminelle Absicht ist eine Form der Straftat geworden. Der Polizei kommt dabei eine bedeutendere Rolle zu. Sie muss für die Prävention von Straftaten verstärkt Informationen und Daten sammeln. Eine große Herausforderung ist damit der Schutz persönlicher Daten und der Datenaustausch zwischen den Ländern. Zweitens wird Verwaltungsrecht wichtiger. Die Normdurchsetzung erfolgt inzwischen in einigen Bereichen, in denen früher das Strafrecht verwendet wurde, durch das Verwaltungsrecht. Schließlich sieht Besselink eine Dynamik zwischen nationalen und internationalen Gerichten, die sich entweder in ihren Urteilen behindern und widersprechen oder ihre Urteile gegenseitig bekräftigen.

Ciril Ribičič trug anschließend zum Thema „Recht auf Privatheit versus öffentliche Sicherheit" vier Fälle des slowenischen Verfassungsgerichts vor. Es handelte sich um Fälle, in denen die slowenische Regierung Waffen in einem geheimen Abkommen nach Israel exportiert hatte, NATO-Flugzeugen die Nutzung ihres Luftraumes erlaubte und der slowenische Geheimdienst Telefonate abgehört hatte. In all diesen Fällen hatten einzelne Bürger Beschwerden beim Verfassungsgericht eingereicht. Das slowenische Verfassungsgericht hat in Urteilen insgesamt den Rechtsstaat sowie die Position der Bürger gestärkt. Im Fall der NATO-Flugzeuge und dem geheimen Abkommen zwischen Israel und Slowenien hat es die Regierung zu weiteren legislativen Maßnahmen aufgefordert, um die Legalität der Handlungen zu gewährleisten. Das Abhören von Telefongesprächen wurde eingeschränkt. Insgesamt hält Ribičič es für notwendig, sich einer Neugestaltung des Verhältnisses zwischen Freiheit und Sicherheit im Lichte der Bedrohungen durch den Terrorismus und durch die organisierte Kriminalität zu verweigern. Rechtsstaatlichkeit ist ein ent- 
scheidendes Element der Gewaltenteilung und damit der Beschränkung der exekutiven Macht. Die Freiheit darf nicht eingeschränkt werden.

Der nächste Vortrag über „Meinungs- und Pressefreiheit versus öffentliche Sicherheit" wurde in Vertretung für Ricardo García Macho, der den Vortrag schrieb, von Jean-François Flauss kurz zusammengefasst. Der Europäische Gerichtshof für Menschenrechte spielte eine entscheidende Rolle beim Schutz der Meinungs- und Pressefreiheit in Europa. In mehreren Urteilen urteilte er zugunsten dieses Menschenrechtes und der Kläger und gegen Staaten (Italien, die Schweiz und Spanien), die in drei Fällen die Meinungs- und Pressefreiheit von Personen aufgrund der öffentlichen Sicherheit einschränkten.

Tania Groppi berichtete im Anschluss über den „Transnationalen Dialog der Gerichte in Anti-Terrorismusfällen“. Dabei fand sie in einer komparativen Analyse heraus, dass die Länder im Bereich der Antiterrorgesetzgebung in einem Dialog miteinander stehen. Durch Präzedenzfälle entsteht eine Konvergenz zwischen den Staaten. Nach dem 11. September 2001 gab es in vielen Staaten ähnliche Entwicklungen im Bereich der Terrorismusbekämpfung, wie eine stärkere Gewichtung der Prävention, das zeitlich unbestimmte Festhalten von Gefangenen ohne Gerichtsurteil, einen Abbau von Prozessrechten für Angeklagte und eine generelle Zunahme der Macht der Exekutive. Gerichte müssen beim Antiterrorkampf mehrere Werte abwägen. Sicherheit ist einer von vielen Werten. Überraschend war, dass viele Gerichte infolge des 11. September den Regierungen Schranken gesetzt haben und damit oftmals aktiv die Bürger- und Menschenrechte verteidigt haben. Je höher die Stellung der Gerichte, desto mutiger waren diese gegenüber den Regierungen in ihren Urteilen. Niedere Gerichte waren zurückhaltender bei der Infragestellung der neuen Antiterrorgesetze. Das erste Urteil nach dem 11. September, welches die Antiterrorgesetzgebung in einem Staat infrage stellte, kam von den Law Lords in Großbritannien. 2002 hat der kanadische Oberste Gerichtshof die Möglichkeit der Regierung, eine Person in ein Land auszuliefern, in dem dieser Person Folter droht, eingeschränkt (aber nicht komplett verboten). Ab 2004 erklärte dann der Oberste Gerichtshof der USA verschiedene Aspekte der amerikanischen Antiterrormaßnahmen für nicht verfassungskonform. Die Gerichte zogen oft in ihren Urteilen Entscheidungen ausländischer Gerichte als Präzedenzfälle hinzu. In den USA gibt es darüber eine lebhafte Debatte, in welcher der oberste Richter Antonin Scalia aus Prinzip nie ausländisches Recht als Entscheidungsgrundlage heranzieht, während der oberste Richter Stephen G. Breyer genau gegenteilig prinzipiell ausländisches Recht als eine Rechtsquelle und damit als eine Entscheidungsgrundlage betrachtet.

Didier Maus fasste schließlich den Tag zusammen und arbeitete dabei als die großen Linien den Begriff der Sicherheit, die Möglichkeiten der Verteidigung der Demokratie und vor allem das Verhältnis zwischen Freiheit und Sicherheit in all seinen Facetten heraus. Ein Beispiel für dieses Verhältnis ist die umstrittene Dauer der Inhaftierung eines Verdächtigen ohne richterliche Prüfung. Wo liegt hier die noch vertretbare Grenze, bei 48 Stunden, zehn Tagen oder gar dreißig Tagen?

Freiheit und Sicherheit in der globalen Sicherheitsarchitektur: die europäische Dimension

Der erste Vortrag wurde von Franz Merli über „Innere Sicherheit als europäische Aufgabe?" gehalten. Drei Aussagen liegen dem Vortrag zugrunde. Erstens, Sicherheit ist eine europäische Aufgabe. Zweitens, die Europäische Union hat viel erreicht im Bereich der Sicherheit. Drittens, aus rechtsstaatlicher Sicht ist die Situation der inneren Sicherheit in Europa unhaltbar. Die Globalisierung des Verbrechens als neue Herausforderung bei der Bekämpfung der Kriminalität hat als logische Konsequenz eine Zusammenarbeit im Sicherheitsbereich auf europäischer Ebene 
zur Folge. Der Bereich der Sicherheit ist eine konkurrierende Zuständigkeit, deren Kompetenz zwischen der Europäischen Union und den Mitgliedstaaten geteilt ist. Die EU-Mitgliedstaaten verfügen primär über die Schutzpflicht gegenüber dem Bürger, falls die Europäische Union keine Zuständigkeit besitzt (wie beim Binnenmarkt). Die Zunahme der Bedeutung der Sicherheitszusammenarbeit zeigt sich unter anderem darin, dass allein 252 Rechtsakte auf europäischer Ebene bestehen, welche die Zusammenarbeit der Polizei zwischen den Mitgliedstaaten regeln. Weiterhin gibt es immer mehr Ausschüsse und Organe, die sich mit dem Thema Sicherheit befassen. In vielen Grenzbereichen gewinnt Sicherheit ebenfalls an Bedeutung, wie beim gemeinsamen Satellitenprojekt Galileo, welches auch zur Grenzüberwachung genutzt werden soll, bei der Pflicht zur Datenspeicherung für Telekommunikationsunternehmen oder der Rückführung von illegalen Fluggästen durch die Fluggesellschaften. Auf europäischer Ebene spielen der Austausch und die zentrale Steuerung von Informationen eine stärker werdende Rolle. Es gibt inzwischen viele Datenbanken der Europäischen Union, zum Beispiel für Visa, Asylbewerber und gesuchte Personen (Schengener Informationssystem SIS). Der europäische Raum der Freiheit, der Sicherheit und des Rechts ist ebenfalls ein gemeinsamer Informationsraum.

Problematisch ist, dass die Zusammenarbeit im Bereich der Sicherheit keinen rechtsstaatlichen Standards genügt. Erstens, EU-Verordnungen, welche Pflichten für Bürger oder Unternehmen beinhalten, müssen grundsätzlich veröffentlicht werden. Das ist nicht immer der Fall. ,Normenklarheit" als rechtsstaatliche Norm ist ebenfalls nicht gegeben. Die Normen sind nicht immer verständlich. Zweitens, der Datenschutz ist nicht ausreichend gewährleistet. Der Empfänger und der Verwendungszweck von gesammelten Daten müssen klar sein. Dieses ist nicht der Fall. Viele Daten werden weltweit ausgetauscht, ohne dass immer deutlich ist, welche Daten für wen zu welchem Zweck bestimmt sind. Die Entschei- dung der Datenerhebung wird oft getrennt von der Entscheidung der Datenverwendung getroffen. Das ist aus rechtsstaatlicher Sicht ein Problem. So werden Daten gesammelt und erst später wird entschieden, was mit diesen Daten geschehen soll. Drittens, der Rechtsschutz ist in der Europäischen Union nicht ausreichend gewährleistet. Zum Beispiel gab es in der Europäischen Union, schwarze Listen' mit Terrorverdächtigen, welche vom Europäischen Gerichtshof für nichtig erklärt wurden, aber die für den Rechtsschutz zuständigen EU-Instanzen waren in diesem Fall alle unterschiedlicher Meinung. Diese Defizite sind unhaltbar geworden und nach Ansicht von Merli müssen die Freiheit, die Achtung der Menschenrechte und der Rechtsstaat in der Europäischen Union unbedingt gestärkt werden.

Der Vortrag von Eleanor Sharpston handelte von der Entstehung des Raumes der Freiheit, der Sicherheit und des Rechts und der Arbeit des Europäischen Gerichtshofes in diesem Bereich. Dieser Raum ist vergleichbar mit der Zusammenarbeit im Binnenmarkt Anfang der 60er-Jahre. Viele Richtungsentscheidungen sind noch nicht getroffen, konkurrierende Prinzipien noch nicht ausbalanciert worden. Die Zusammenarbeit im Rechts- und Sicherheitsbereich entstand aus der Notwendigkeit die organisierte Kriminalität, welche die neue Freizügigkeit innerhalb der Europäischen Union für sich nutzte, effektiver bekämpfen zu können. Insgesamt bestand dabei eine große Zurückhaltung der Mitgliedstaaten ihre Rechtssysteme zu harmonisieren, da jeder sein System naturgemäß für das beste hält. Die Lösung bestand in der gegenseitigen Anerkennung der Rechtsakte der fremden Rechtssysteme und der Schaffung des Raumes der Freiheit, der Sicherheit und des Rechts und seiner Instrumente (wie zum Beispiel des europäischen Haftbefehls). Dabei ist erstaunlich, dass die Mitgliedstaaten, die ihren Rechtssystemen mehr vertrauen als fremden Systemen, mindestens genauso viel Vertrauen in die fremden Rechtssysteme benötigen beim Verfahren der gegenseitigen 
Anerkennung fremder Urteile und Gesetze wie bei einer möglichen Harmonisierung der Systeme. Immer mehr Bereiche der dritten Säule wurden in die erste Säule transferiert. Das hat Auswirkungen auf die Bürger und Angehörige anderer Staaten (zum Beispiel Asylrecht). Die Arbeit des Europäischen Gerichtshofes ist für die Bürger wichtig, da viele verabschiedete EU-Verordnungen ambivalente und unklare Formulierungen enthalten und damit schlechte Verordnungen sind. Das erklärt sich aus der Notwendigkeit auf der europäischen Ebene Kompromisse zwischen den Mitgliedstaaten zu finden. Das Problem ist aber, dass diese Texte die Bürger der Europäischen Union betreffen und aufgrund der ambivalenten Formulierungen muss der Europäische Gerichtshof (EuGH) in vielen Fällen die Texte verbindlich interpretieren. Hierbei spielt es eine große Rolle, ob eine Verordnung unter die erste oder dritte Säule der Europäischen Union fällt. Der EuGH würde es im Übrigen bevorzugen, wenn die Mitgliedstaaten auf der EU-Ebene stärker legislativ tätig würden und es nicht dem EuGH überlassen würden, auf der Grundlage existierender Prinzipien von den Mitgliedstaaten nicht geregelte Probleme zu entscheiden. Die Mitgliedstaaten besitzen die Legitimität für die legislative Tätigkeit, nicht der EuGH. Das ganze Verhältnis zwischen der Europäischen Union, der Europäischen Menschenrechtskonvention, dem internationalen Völkerrecht und nationalem Verfassungsrecht zum Schutz der Unionsbürger ist insgesamt noch nicht austariert (welche Norm wird wie und wo angewandt und genießt wann Priorität über andere Normen).

Péter Kovács referierte in seinem Vortrag über die Einwände nationaler Gerichte zum europäischen Haftbefehl. Der Europäische Rat führte den europäischen Haftbefehl durch mehrere Beschlüsse seit 2002 ein. 2006 wurde der europäische Haftbefehl auf Island und Norwegen übertragen. Das Ziel war eine Vereinfachung und Beschleunigung der Auslieferung von Verdächtigen ohne politische Einmischung. Der Haftbefehl kann für 32
Verbrechen ausgestellt werden, wie zum Beispiel Mitgliedschaft in einer kriminellen Organisation, Terrorismus, Menschenhandel, sexuelle Ausbeutung Minderjähriger, Mord, schwere Köperverletzung, Korruption oder Produktfälschung. Die Staaten einigten sich darauf, weder ein politisches Motiv für ein Verbrechen noch die Nationalität des Straftäters als Entschuldigung für die Verweigerung der Auslieferung zu verwenden. Jeder Staat muss einen Gesuchten ausliefern, wenn er sich auf seinem Territorium befindet und keine doppelte Bestrafung (Prinzip , ne bis in idem') zu befürchten ist. Gegen den europäischen Haftbefehl gab es infolge seiner Einführung mehrere Klagen vor nationalen Verfassungsgerichten und obersten Gerichten. In Frankreich, Polen, Deutschland, Zypern, Portugal, Tschechien und Slowenien wurde die Verfassungsmäßigkeit des Haftbefehls von den jeweiligen Gerichten diskutiert und teilweise verneint. So muss in Polen die Verfassung geändert werden, um die Auslieferung polnischer Staatsbürger zu ermöglichen. Das mögliche Problem der doppelten Strafbarkeit durch den europäischen Haftbefehl wurde von Verfassungsgerichten in Frankreich, Tschechien, Belgien und Ungarn diskutiert. Insgesamt war der europäische Haftbefehl damit Gegenstand vielfältiger juristischer Kontroversen.

Freiheit und Sicherheit in der globalen Sicherheitsarchitektur: die internationale Dimension

Lauri Mälksoo hielt den Einführungsvortrag, in welchem er einen allgemeinen Überblick über die Entwicklung des Völkerrechts gab. Dabei konstatierte er, dass es eine Vielzahl internationaler Verträge gibt. Das Völkerrecht entwickelte sich, weil das Naturrecht zu vage war. Die wirkliche Universalisierung des internationalen Rechts begann erst nach dem Zweiten Weltkrieg. Vorher war das internationale Recht europäisch. Ein grundlegendes Dilemma des internationalen Rechts in seiner Reaktion auf Bedrohungen wie al-Qaida ist die Tatsache, dass das internationale Recht als 
reziprokes Recht ausgestaltet wurde. AlQaida stellt eine neue Dimension der Bedrohung dar, welche nicht in dieses ursprüngliche Schema der Reziprozität passt. Deshalb wurde die Gültigkeit des internationalen Rechts von der amerikanischen Bush-Administration teilweise infrage gestellt.

Xavier Philippe sprach dann über den ,verfassungsrechtlichen Rahmen für Akte der Armee und der Sicherheitskräfte im Falle der Gefährdung der internationalen Sicherheit". In den meisten Ländern entscheidet die Exekutive über den Einsatz der Streitkräfte im Ausland. In Frankreich hat der Staatspräsident ebenfalls dieses Vorrecht. Allerdings wurde der Artikel 35 der französischen Verfassung 2008 geändert und hat dem Parlament wichtige Rechte beim Einsatz der Armee im Ausland eingeräumt. So darf das Parlament einen Rückzug der Soldaten beschließen und das Parlament hat das Recht einen Krieg zu erklären beziehungsweise muss den Einsatz von Soldaten im Ausland billigen, falls dieser nach Ablauf von vier Monaten noch andauert. Ein Problem dieses Artikels ist, dass er nicht auf militärische Operationen angewendet werden kann, die außerhalb des Territoriums eines fremden Staates stattfinden (Beispiel Operation gegen die Piraten am Horn von Afrika). Die Rechte des Parlaments über den Einsatz der Streitkräfte im Ausland zu entscheiden variieren von Land $\mathrm{zu}$ Land. Am meisten Rechte hat der Deutsche Bundestag, der jeden Einsatz deutscher Soldaten im Ausland genehmigen muss. In Italien muss das Parlament nicht über den Einsatz entscheiden. Allerdings muss es über die Finanzierung eines Einsatzes von Soldaten befinden und somit ist eine indirekte Zustimmung notwendig. In Großbritannien sucht die Regierung in der Regel die Unterstützung des Parlaments. Sie ist aber nicht zwingend. In den USA regelt der „War Powers Act“ von 1973 die Rechte des Kongresses beim Einsatz der Streitkräfte im Ausland. Der Präsident verfügt über weitreichende Vollmachten beim Einsatz der Streitkräfte.

Heike Krieger trug anschließend zur „Haltung europäischer Gerichte in Sicherheitsfragen“ vor. Insgesamt kam Krieger zu dem Schluss, dass die Gerichte eher zurückhaltend in Sicherheitsfragen agieren und der Exekutive und der Legislative einen weiten Spielraum bei der Beurteilung von Bedrohungen der Sicherheit einräumen. So hat zum Beispiel der Europäische Gerichtshof für Menschenrechte der Türkei und Großbritannien (Nordirlandkonflikt) einen weiten Ermessensspielraum bei der Bekämpfung von Bedrohungen der inneren Sicherheit gewährt. Heutzutage sind die Gerichte deutlich kritischer in ihren Entscheidungen zur Antiterrorgesetzgebung als dies in den 70er-Jahren der Fall war. Damals haben die Gerichte die politischen Entscheidungen weitgehend akzeptiert.

Dieter Scheuing hat die Tagung abschließend zusammengefasst. Das Verhältnis zwischen Freiheit, Sicherheit und dem Rechtsstaat muss permanent neu austariert werden. Es befindet sich nie in einer Balance. Eine dieser neuen Austarierungen findet momentan im Lichte neuer Bedrohungen statt. Beim Spannungsfeld zwischen individuellem Rechtsschutz und Sicherheit müssen die individuellen Rechte wieder stärker respektiert werden. Beim Kampf gegen den Terrorismus müssen die Menschenrechte beachtet werden. Der Rechtsschutz auf der internationalen Ebene ist unzureichend und in der Europäischen Union nicht perfekt. In beiden Bereichen sollte mehr getan werden, um den individuellen Rechtsschutz besser gewährleisten zu können. 